\title{
Oxidative stress and breast cancer biomarkers: the case of the cytochrome P450 2E1
}

\author{
Subir Singh ${ }^{1}$, Ramkumar Rajendran², Kengo Kuroda ${ }^{3}$, Emiko Isogai ${ }^{3}$, Marija Krstic-Demonacos ${ }^{4}$, \\ Constantinos Demonacos ${ }^{1}$ \\ ${ }^{\prime}$ Manchester Pharmacy School, University of Manchester, Manchester M13 9PT, UK. \\ ${ }^{2}$ School of Pharmacy, International Medical University, Bukit Jalil, 57000 Kuala Lumpur, Malaysia. \\ ${ }^{3}$ Laboratory of Animal Microbiology, Tohoku University Graduate School of Agricultural Science, Sendai, Miyagi, 981-8555, Japan. \\ ${ }^{4}$ School of Environment and Life Sciences, University of Salford, Peel Building, Salford, M5 4WT, UK.
}

Correspondence to: Dr. Constantinos Demonacos, Manchester Pharmacy School, University of Manchester, Stopford building, Oxford Road, Manchester M13 9PT, UK. E-mail: cdemonacos@manchester.ac.uk

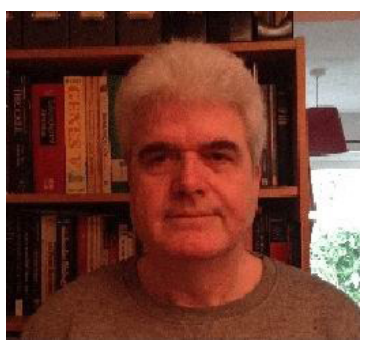

Dr. Demonacos joined the University of Manchester, Manchester Pharmacy School in 2003 where he is involved in the investigation of the role of ROS in cellular energy metabolism and breast carcinogenesis. In addition, Dr. Demonacos' laboratory explores the signaling events that facilitate cancer cells to evade immunosurveillance.

\begin{abstract}
A B S T R A C T
Aim: The aim of the study is to investigate the impact of the cytochrome P450 2E1, which is the most efficient CYP450 family member in generating reactive oxygen species (ROS), on cellular energy metabolism of breast cancer cells and therefore the effects of CYP2E1 on breast carcinogenesis. Methods: The estrogen receptor positive MCF-7 and the triple negative MDAMB-231 breast cancer cells were used as experimental system to estimate ROS generation in these cells overexpressing CYP2E1 and treated with the glycolytic inhibitors 3-bromopyruvate or 2-deoxyglucose in the presence or absence of the CYP2E1 inhibitor chlormethiazole. Adenosine triphosphate (ATP) assay was used to measure ATP production and lactate assay to quantify the efflux of lactic acid in breast cancer cells treated with the CYP2E1 inhibitor chlormethiazole, the mitochondrial membrane potential and cell viability assays were employed to assess the pathway of cellular energy production and cellular death respectively after treatment of MCF-7 and MDA-MB-231 with the CYP2E1 activator acetaminophen or the CYP2E1 inhibitor chlormethiazole. Results: The results indicated increased ROS generation in breast cancer cells overexpressing CYP2E1. ROS generation was differentially regulated in breast cancer cells upon treatment with the CYP2E1 inhibitor chlormethiazole. Chlormethiazole treated MCF-7 cells exhibited reduced lactate efflux implying that CYP2E1 directly or indirectly regulates the glycolytic rate in these cells. Furthermore the mitochondrial membrane potential of both MCF-7 and MDA-MB-231 cells was differentially affected by the CYP2E1 activator acetaminophen versus the CYP2E1 inhibitor chlormethiazole providing additional support for the involvement of CYP2E1 in energy metabolic pathways in breast cancer. Conclusion: Results presented in this study provide evidence to suggest that CYP2E1 regulates cellular energy metabolism of breast cancer cells in a manner dependent on cell type and potentially on the clinical staging of the disease therefore CYP2E1 is a possible breast cancer biomarker.
\end{abstract}

Key words: Reactive oxygen species; cytochrome P450 2E1; glycolysis; breast cancer

\section{INTRODUCTION}

Reactive oxygen species (ROS) such as superoxide, hydroxyl radical, and hydrogen peroxide are metabolic by-products leaking from the complexes I and III of the mitochondrial respiratory chain. ${ }^{[1]}$ Generation of high ROS levels is

\begin{tabular}{|l|l|}
\hline \multicolumn{3}{|c|}{ Access this article online } \\
\hline Quick Response Code: & Website: \\
& http://www.jcmtjournal.com \\
\cline { 2 - 2 } & \\
\hline
\end{tabular}

detrimental for the cells as it can lead to DNA damage and oxidation of proteins and lipids changing their functions. ${ }^{[2]}$ Accumulating evidence indicates that apart from their

This is an open access article distributed under the terms of the Creative Commons Attribution-NonCommercial-ShareAlike 3.0 License, which allows others to remix, tweak, and build upon the work non-commercially, as long as the author is credited and the new creations are licensed under the identical terms.

For reprints contact: service@oaepublish.com

How to cite this article: Singh S, Rajendran R, Kuroda K, Isogai E, Krstic-Demonacos M, Demonacos C. Oxidative stress and breast cancer biomarkers: the case of the cytochrome P450 2E1. J Cancer Metasta Treat 2016;2:268-76.

Received: 24-06-2016; Accepted: 23-07-2016. 
harmful effects ROS act as second messenger signalling molecules regulating numerous pathways including cell cycle, ${ }^{[3]}$ autophagy, ${ }^{[4,5]}$ apoptosis, ${ }^{[6]}$ endoplasmic reticulum (ER) stress $^{[7]}$ and cellular energy metabolism. ${ }^{[8,9]}$

Sources of intracellular ROS generation include both organelles such as mitochondria, ER and peroxisomes as well as enzymes such as the NADPH oxidases, xanthine oxidase, lipoxygenases and cytochrome P450 enzymes, which produce ROS through their enzymatic activities. ${ }^{[10]}$ CYP450 enzymes are mainly involved in the phase I metabolism of a wide range of exogenous and endogenous compounds oxidizing them to form more hydrophilic molecules thereby facilitating easier clearance. ${ }^{[11]}$ In the case the monooxygenation reaction catalysed by CYP enzymes is uncoupled from the NADPH reaction instead of a monooxygenated substrate production of ROS occurs. ${ }^{[12]}$ The CYP450 family member CYP2E1 is the most active enzyme of the family in terms of generating ROS sometimes inducing production of oxygen radicals even in the absence of substrates. ${ }^{[13]}$

Apart from the liver CYP2E1 gene expression has been detected in other tissues such as breast, lung, kidney and hematopoietic tissues ${ }^{[13]}$ and has been reported to be over expressed in malignant compared to normal tissues. ${ }^{[14-17]}$ CYP2E1 overexpression in cancer is attributed to the inflammatory conditions present in the tumor microenvironment characterised by increased inflammatory cytokine production which affects CYP2E1 gene expression. ${ }^{[18-20]}$ Several CYP2E1 dependent mechanisms contributing to tumorigenesis have been suggested including formation of toxic intermediate derivatives and activated carcinogens. ${ }^{[21-23]}$ CYP2E1 mediated ROS generation could also contribute to tumor development through pathways in which ROS play vital role such as DNA damage, enhanced angiogenic responses $^{[24]}$ autophagy ${ }^{[4,25,26]}$ ER stress $^{[27]}$ and unfolded protein response (UPR). ${ }^{[28]}$ Furthermore, research in our laboratory has indicated that CYP2E1 is differentially expressed in a manner dependent on the genetic background and the stage of breast cancer, regulating oxidative stress response and metastasis. ${ }^{[2]]}$

Cancer cells produce energy predominantly through aerobic glycolysis -- a phenomenon also called Warburg effect -- rather than oxidative phosphorylation even in the presence of oxygen and functional mitochondria. ${ }^{[30]}$ The Warburg effect is induced in cancer cells by increased cellular glucose uptake stimulated by ROS mediated upregulation of gene expression of glucose transporters such as GLUT-1. ${ }^{[31]}$ On the other hand, experimental evidence supports the view that increased glycolytic conversion to pyruvate leads to ROS generation ${ }^{[32]}$ suggesting the existence of an interrelation between ROS generation with glycolysis and vice versa. ${ }^{[9,33]}$ the hypothesis that overexpression of CYP2E1 and the resultant elevated ROS production might regulate cellular energy metabolism in cancer cells pointing out CYP2E1 as a potential cancer biomarker. The understanding of the interplay between CYP2E1 -- ROS generation -- cellular energy metabolism can provide important conclusions towards establishing novel breast cancer biomarkers and overcoming drug resistance. The estrogen receptorpositive MCF-7 and the triple negative MDA-MB-231 [estrogen receptor-negative, progesterone receptornegative and human epidermal growth factor receptor 2 (HER2)-negative] breast cancer cells were used in this study to evaluate the impact of the CYP2E1 mediated ROS generation on the energy metabolism of these cells.

\section{METHODS}

\section{Cell culture}

The human breast carcinoma cell lines MCF-7 and MDAMB-231 [obtained from the European Collection of Cell Cultures (ECACC)] were maintained in Dulbecco's modified Eagle's medium (Sigma-Aldrich, Gillingham, UK), supplemented with $10 \%$ foetal bovine serum (Gibco, Paisley, UK) and $1 \%$ penicillin/streptomycin (Lonza, Allendale, NJ, USA) at $37^{\circ} \mathrm{C}$ in a humidified atmosphere containing $5 \% \mathrm{CO}_{2}$. Cells were treated with $100 \mu \mathrm{M}$ 3-bromopyruvate (3BP) (Sigma-Aldrich) for $3 \mathrm{~h}, 20$ $\mathrm{mmol} / \mathrm{L} 2$-deoxyglucose (2DG) (Sigma-Aldrich) for $24 \mathrm{~h}$, $2.5 \mathrm{mmol} / \mathrm{L}$ acetaminophen (APAP) (Sigma-Aldrich) for $3 \mathrm{~h}$ and $20 \mu \mathrm{M}$ chlormethiazole (CMZ) (Sigma-Aldrich) for $16 \mathrm{~h}$.

\section{Transient transfection}

Transient transfections were carried out using the polyfect transfection reagent (Qiagen, Crawley, UK), according to the manufacturer's instructions. Constructs used for ectopic expression included the pcDNA ${ }^{\mathrm{TM}} 3.1$ (Invitrogen) and the pCI-neo-CYP2E1 (kindly provided by Dr. Cederbaum, Mount Sinai School of Medicine, New York). ${ }^{[29]}$

\section{Measurement of ROS}

Cells were grown until they reached $80 \%$ confluence prior to transient transfection and different treatments. ROS levels were measured using flow cytometry as described previously. ${ }^{[29]}$ Cells were transiently transfected with the indicated constructs and $16 \mathrm{~h}$ after transfection they were harvested and incubated with $1 \mathrm{~mL}$ of APCH7-conjugated CD20 antibody (BD Biosciences, Franklin Lakes, NJ, USA) to detect only the cells ectopically expressing CYP2E1. Cells were then incubated with H2DCFDA (Invitrogen, Carlsbad CA, USA) in the dark at $37^{\circ} \mathrm{C}$ for $30 \mathrm{~min}$ and subjected to flow cytometry using CYAN-ADP flow cytometer (Dako, Glostrup, Denmark) following the fluorescence profile of the H2DCFDA and APC-H7 probes.

\section{Adenosine triphosphate (ATP) assay}

ATP levels were measured using the ViaLight plus kit

Taken together, the above mentioned observations allow 
(Lonza, Slough, UK), based on the bioluminescent measurement of ATP present in cells. ATP monitoring reagent (AMR plus) was prepared by adding assay buffer into the vial containing the lyophilized AMR and incubated at room temperature for $15 \mathrm{~min}$ for complete rehydration. Cells were lysed in $50 \mu \mathrm{L}$ of cell lysis reagent for $10 \mathrm{~min}$. A total volume of $100 \mu \mathrm{L}$ of cell lysate was added to a luminometer plate and $100 \mu \mathrm{L}$ of AMR plus was added to the appropriate well. The plate was then incubated at room temperature for $2 \mathrm{~min}$ and values were obtained from the luminometer.

\section{Lactate assay}

To measure the lactate efflux MCF-7 and MDA-MB-231 breast cancer cells were grown in 6 well plates and left untreated or treated with CYP2E1 specific inhibitor CMZ.
Media was collected in a 96 well plates after treatment. Two microlitre of this media was mixed with $60 \mu \mathrm{L}$ of lactate reagent and incubated at room temperature for $15 \mathrm{~min}$ and the absorbance was recorded at $540 \mathrm{~nm}$. Lactic acid standard solutions (Trinity Biotech, Ireland) were used to plot the standard curve and the concentration of lactic acid present in the media was calculated accordingly. Lactate production rates were expressed as $\mathrm{mmol} / \mathrm{L}$.

\section{Mitochondrial membrane potential}

Mitochondrial transmembrane potential $(\Delta \psi \mathrm{m})$ was measured using the cationic dye JC- $1(5,5,6$, 6-tetrachloro-1,1,3,3-tetraethylbenzimidazolcarbocyanine iodide) (ChemoMetec, Allerod, Denmark) using the NucleoCounter ${ }^{\circledR}$ NC-3000 ${ }^{\mathrm{TM}}$ system. Cells were grown in 6-well plates and treated with the CYP2E1 activator APAP
(A)

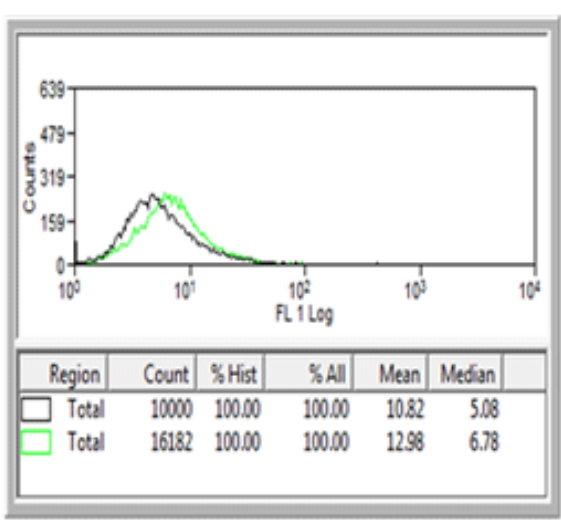

MDA-MB-231

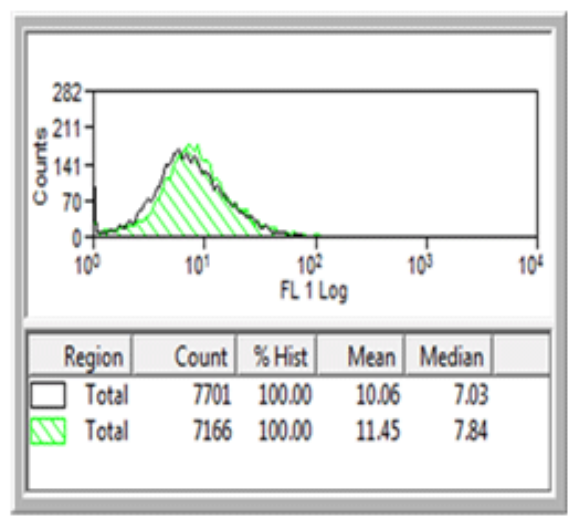

\section{pcDNA3}

CYP2E1

(B)
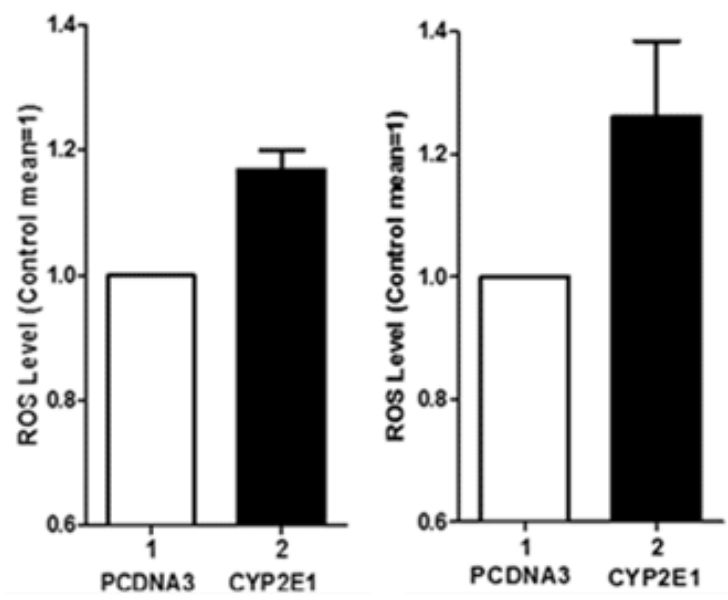

Figure 1: ROS generation in MCF-7 and MDA-MB-231 cells ectopically expressing CYP2E1. MCF-7 and MDA-MB-231 cells were transiently transfected with a CYP2E1 expressing or the empty vector PCDNA3. ROS levels were determined using H2DCFDA fluorescent dye and flow cytometry only in the cells ectopically expressing CYP2E1 (co-transfected with CD20). FACS data were analyzed using Beckman Coulter Summit 4.1 software. (A) Histograms displaying ROS levels after transient transfection of CYP2E1 or pcDNA3 as indicated. Green coloured histograms represent ROS levels in cells transfected with CYP2E1 and black histograms represent ROS levels in cells transfected with PCDNA3; (B) bar graphs representing ROS levels generated in cells transfected with PCDNA3 and CYP2E1 as indicated. Data are average of three independent experiments. ROS: reactive oxygen species; CYP: cytochrome P450 
A

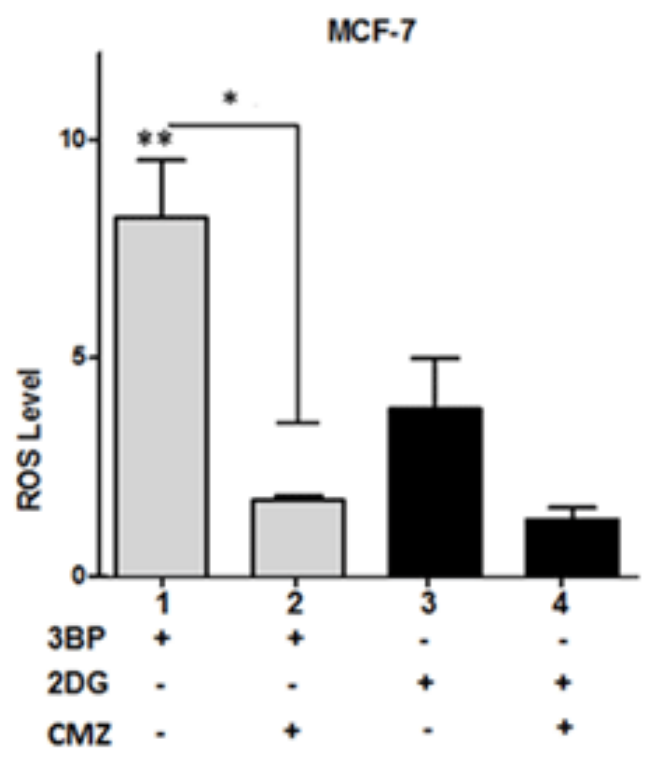

B

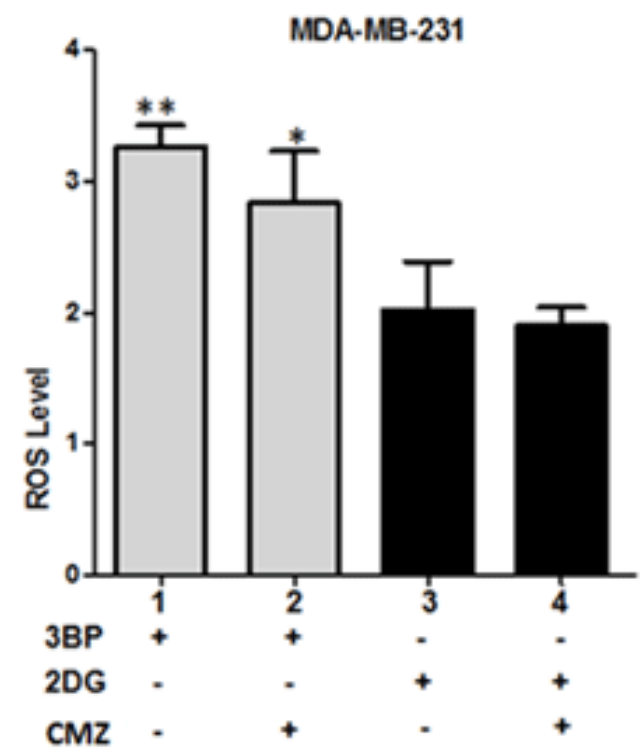

Figure 2: CYP2E1 mediated ROS generation in breast cancer cells under diverse stress conditions. Graph indicating ROS levels generated in 3BP, 2DG and CMZ treated MCF-7 (A) and MDA-MB-231 (B) cells. Error bars represent mean \pm SEM from three independent experiments. Statistical analysis was performed by one-way ANOVA followed by Tukey post hoc for multiple pair-wise comparisons. One asterisk indicates $P<0.05$ and two asterisks $P<0.005$. ROS: reactive oxygen species; CMZ: chlormethiazole

A

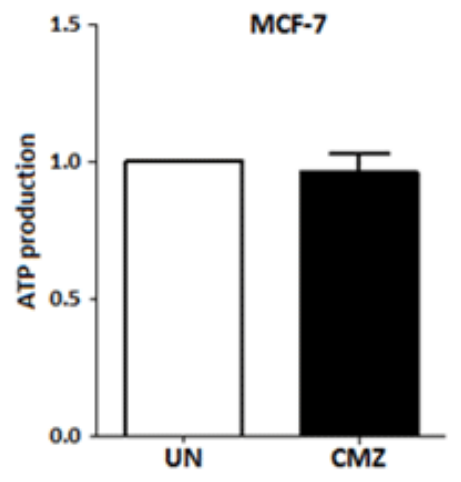

C

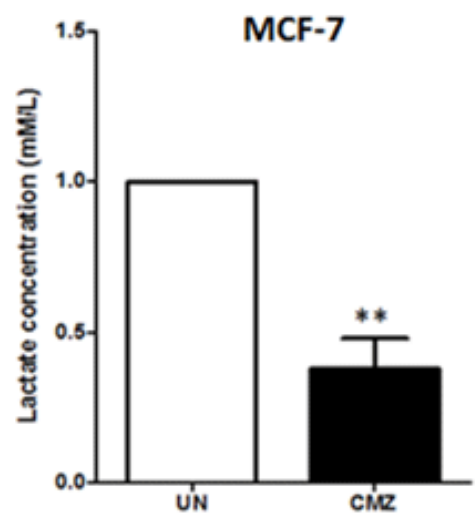

B

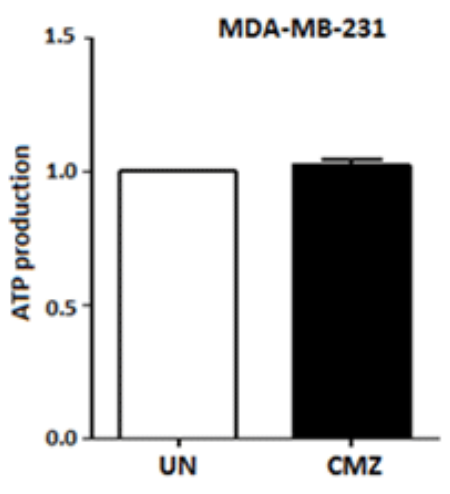

D

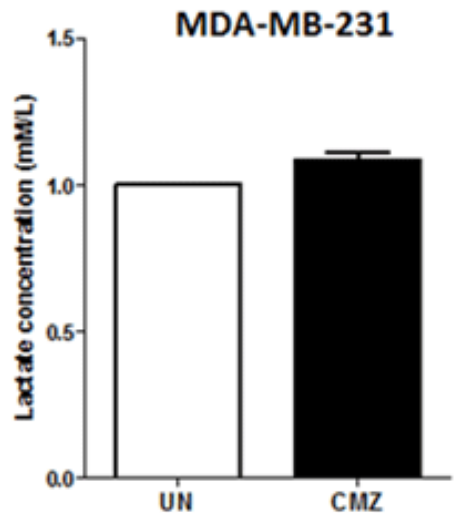

Figure 3: ATP production and lactate efflux in MCF-7 and MDA-MB-231 cells treated with the CYP2E1 inhibitor CMZ. MCF-7 and MDA-MB-231 cells were either left untreated or treated with the CYP2E1 specific inhibitor CMZ. ATP production (A and B) was determined using the ViaLight ${ }^{\mathrm{TM}}$ plus kit (Lonza, Slough, UK) and lactate efflux (C and D) using the lactate reagent (Trinity Biotech, Dublin, Ireland). Data are average of three independent experiments \pm SEM; ${ }^{* *} P<0.005$. ATP: adenosine triphosphate; CMZ: chlormethiazole 
(A)

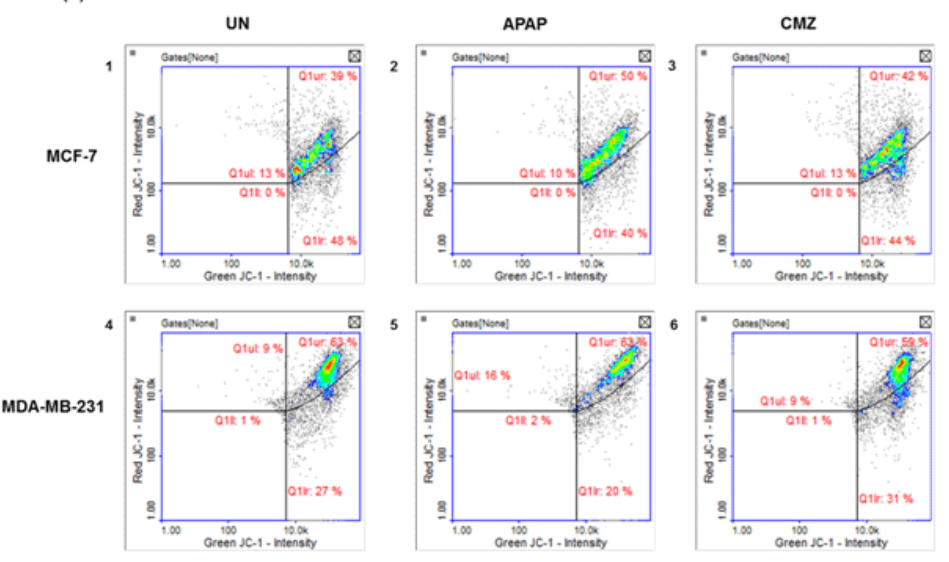

(B) $\quad$ ㅁ MCF-7

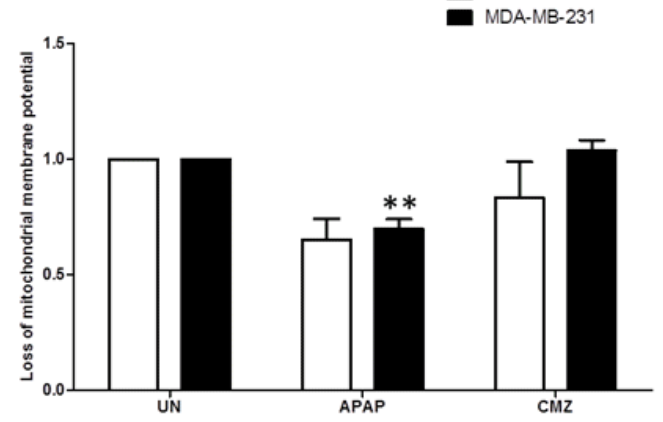

Figure 4: Mitochondrial membrane potential $(\Delta \psi)$ in breast cancer cells treated with the CYP2E1 activator APAP or the CYP2E1 inhibitor CMZ. Breast cancer cells were left untreated or treated with either the CYP2E1 inducer (APAP) or the CYP2E1 inhibitor (CMZ). Mitochondrial membrane potential $(\Delta \psi)$ was determined using JC-1 and DAPI fluorescent dye (ChemoMetec, Allerod, Denmark) and the NucleoCounter NC3000. Data were analyzed using NucleoView software. (A) Histograms representing the mitochondrial membrane potential $(\Delta \psi)$ in breast cancer cells under different stress conditions; (B) bar graphs representing the effect of APAP and CMZ treatments on mitochondrial membrane potential $(\Delta \psi)$ in breast cancer cells. Error bars represent mean \pm SEM from three independent experiments. Two asterisks indicate $P<0.005$. APAP: acetaminophen; CMZ: chlormethiazole

or the CYP2E1 inhibitor CMZ. After treatment, cells were stained with JC-1 and DAPI (ChemoMetec, Allerod, Denmark). Cellular JC-1 monomers and aggregates are detected as green and red fluorescence, respectively. Mitochondrial depolarization and apoptosis are revealed as a decrease in the red/green fluorescence intensity ratio. Necrotic and late apoptotic cells are detected as blue fluorescent (DAPI) cells. After staining cells were loaded on an 8-chamber NC-Slide A $8^{\mathrm{TM}}$ and samples were analysed using the $\mathrm{NC}-3000^{\mathrm{TM}}$ system and the amount of blue, green and red fluorescence of the individual cells was quantified.

\section{Cell viability assay}

Cell viability was measured using the NucleoCounter ${ }^{\circledR}$ NC-3000 ${ }^{\mathrm{TM}}$ system. Cell viability assay was used to detect changes in the intracellular level of (reduced) thiols. Cells were seeded in 6 well plates and cultured until they reached $80 \%$ confluence prior to different treatments. After the treatments, cell culture medium was aspirated and $500 \mu \mathrm{L}$ of cell dissociation buffer was added to cells for dissociation from culture plates. Five hundred microlitre of complete culture medium was added to quench the toxicity of dissociation buffer after cell dissociation.
Then cells were stained with solution 5 as described by the manufacturer. Solution 5 (ChemoMetec, Allerod, Denmark) contains three different stains, each one of them staining either all nucleated cells (DAPI), dead cells (Propidium iodide) or viable cells (VB-48) (ChemoMetec, Allerod, Denmark) and the intensity of the stain depends on the GSH level. After staining, cells were loaded into an 8 -chanmber NC-slide. Samples were analysed using the $\mathrm{NC}-3000^{\mathrm{TM}}$ system.

\section{RESULTS}

The role of CYP2E1 in ROS generation in breast cancer cells has been investigated by our and other groups indicating that overexpression of this cytochrome P450 family member in breast cancer cells coincides with elevated ROS levels implying that CYP2E1 is one of the intracellular sources of ROS. ${ }^{[29,34]}$ To confirm that this is the case in the triple positive MCF-7 and the triple negative MDA-MB-231 cells CYP2E1 expressing vectors were transiently transfected and the ROS levels in mock and ectopically expressing CYP2E1 cells were followed as described in Materials and Methods. Increased ROS levels were recorded in both cell lines ectopically expressing 
(A)

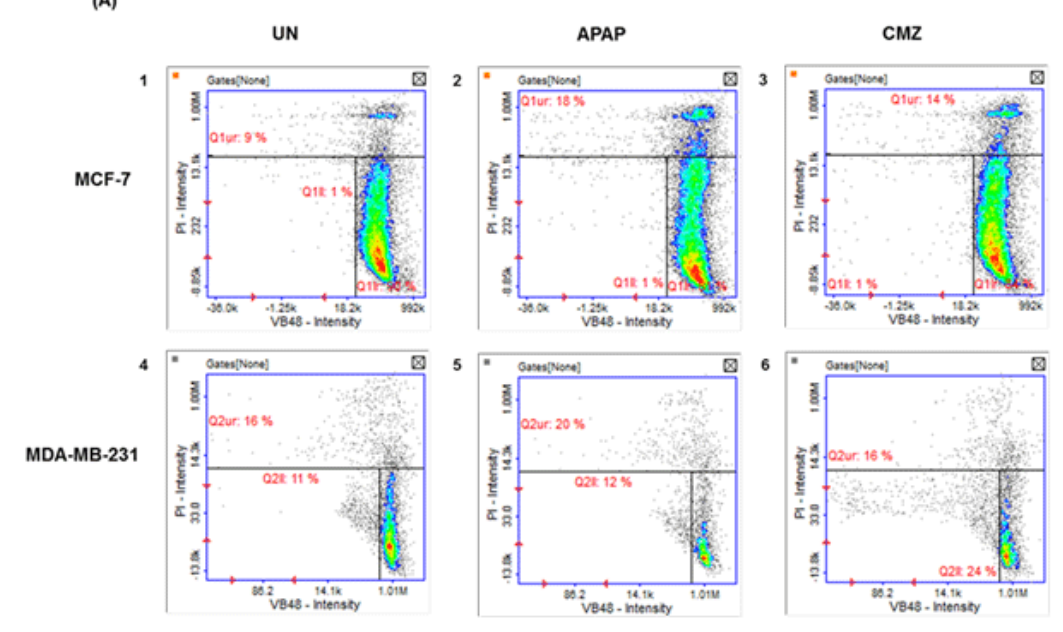

(B)

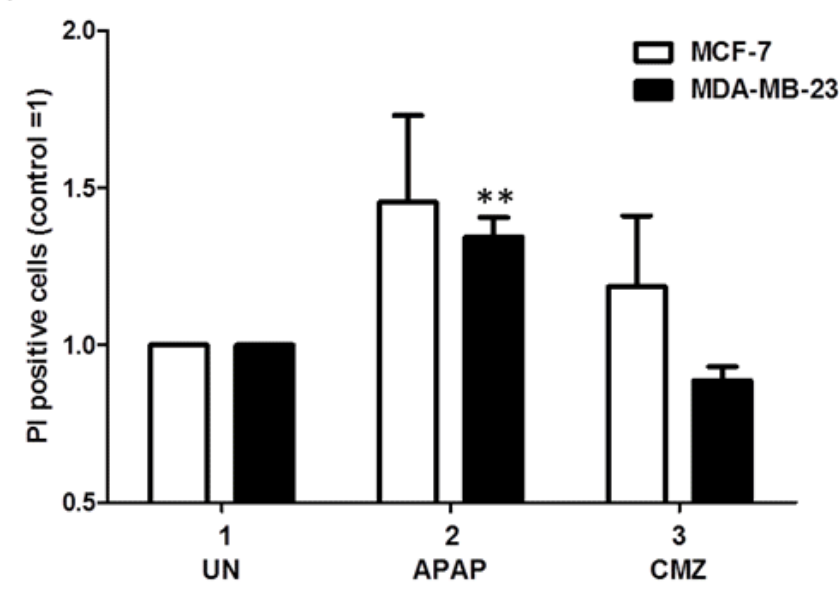

Figure 5: Cell viability of breast cancer cells treated with the CYP2E1 activator APAP or the CYP2E1 inhibitor CMZ. Breast cancer cells were left untreated or treated with either the CYP2E1 activator APAP or the CYP2E1 inhibitor CMZ as indicated. Cell viability was calculated using the Vitality kit Assay (ChemoMetec, Allerod, Denmark). (A) Histogram representing cell viability under different stress conditions. Dead cells stained with PI are shown in the Q1ur gates; (B) bar graph representing the PI positive breast cancer cells treated with either APAP or CMZ. Error bars represent mean \pm SEM from three independent experiments. Two asterisks indicate $P<0.005$. APAP: acetaminophen; CMZ: chlormethiazole

CYP2E1 compared to mock transfected cells [Figure 1B, compare bars 2 to bars 1 respectively].

To explore further the effects of CYP2E1 on the glycolytic pathway of energy production the glycolytic inhibitors 3BP and 2DG were used to inhibit glycolysis in MCF-7 and MDA-MB-231 cells either individually or in combination with the CYP2E1 inhibitor CMZ and the ROS generated under these conditions were monitored as described in Materials and Methods. Treatment of MCF-7 cells with 3BP generated higher ROS levels compared to MCF-7 cells treated with 2DG [Figure 2A, compare bar 1 to bar 3]. Combination of 3BP or 2DG treatment with $\mathrm{CMZ}$ resulted in dramatic decrease of ROS levels in MCF-7 cells [Figure 2A, compare bar 2 to bar 1 and bar 4 to bar 3]. In contrast, in MDA-MB-231 cells CMZ had marginal effect on that observed when cells were treated with the glycolytic inhibitors 3BP and 2DG alone [Figure 2B, compare bars 2 and 4 to bars 1 and 3 respectively] providing additional evidence that CYP2E1 exerts cell type dependent effects in ROS generation in a manner dependent on the genetic background and potentially their invasive potential.

Accumulating evidence supports the notion that ROS generation is associated with cellular energy production. ${ }^{[31,32,35]}$ Results shown in Figure 1 indicate that CYP2E1 overexpression led to elevation of ROS in MCF-7 and MDA-MB-231 breast cancer cells implying a potential role of CYP2E1 in cellular energy metabolism. To test this hypothesis MCF-7 and MDA-MB-231 cells were treated with the CYP2E1 inhibitor CMZ and the levels of ATP produced under these conditions were determined as described in Methods. CMZ treatment of both MCF-7 and MDA-MB-231 cells did not have any significant effect on the ATP produced under these conditions [Figure $3 \mathrm{~A}$ and 3B]. To test whether the ROS levels' profile observed in breast cancer cells was related to lactate production, MCF7 and MDA-MB-231 cells were treated with the CYP2E1 
inhibitor $\mathrm{CMZ}^{[36,37]}$ and lactate production was monitored as described in Methods. Results shown in Figure 3C indicate that inhibition of CYP2E1 in MCF-7 cells resulted in reduction of lactate production in these cells whereas inhibition of CYP2E1 in MDA-MB-231 cells did not have any effect on lactate production [Figure 3D] reiterating the concept that CYP2E1 effects are cell type dependent.

It is known that oxidative stress can trigger the mitochondrial permeability transition and $\Delta \psi$ collapse leading to defects in ATP production. ${ }^{[38]}$ Taking into account these observations, we next assessed potential changes in the mitochondrial membrane potential in breast cancer cells treated with the CYP2E1 activator APAP or the CYP2E1 inhibitor CMZ. APAP treatment of both MCF-7 and MDA-MB-231 cells led to decrease of $\Delta \psi$ compared to untreated cells [Figure 4B, compare APAP white $(0.652)$ and black $(0.698)$ bars to UN white (1) and black (1) bars]. In contrast, CMZ treatment did not exert any effects on $\Delta \psi$ which remained the same as that observed in the untreated cells [Figure 4B, compare CMZ white and black bars to UN white and black bars]. These results indicate a potential role of CYP2E1 mediated ROS generation in the process of alterations of mitochondrial membrane potential.

Alterations in $\Delta \psi$ might in some cases lead to cell death ${ }^{[39]}$ and in order to explore whether that was the case in breast cancer cells treated with APAP or CMZ MCF-7 and MDA-MB-231 cell death was determined by PI staining upon treatment with APAP or CMZ. APAP treatment of MCF-7 and MDA-MB-231 cells led to increased cell death in both cell lines (MCF-7 cells from 9\% to $18 \%$ and MDA-MB-231 cells from 16\% to 20\%) [Figure 5B, compare APAP white and black bars to UN white and black bars]. CMZ treatment of both MCF-7 and MDAMB-231 cells did not exert any effects on cell death as it did not affect the percentage of PI positive compared to untreated MCF-7 and MDA-MB-231 cells [Figure 5B, compare $\mathrm{CMZ}$ white and black bars to UN white and black bars]. Taken together these results indicate that at least in part CYP2E1 mediated generation of ROS alters collapse of mitochondrial membrane potential determining cell survival or death in a cell type dependent manner.

\section{DISCUSSION}

Accumulating evidence supports the view that ROS generated by CYP2E1 activity mediate cell signalling events that promote alterations in the cellular physiology and disease development. ${ }^{[0-43]}$ Studies in our and other laboratories have indicated diverse levels of CYP2E1 gene expression in a manner dependent on the genetic background and the migratory potential of these cells. ${ }^{[16,17,29,34]}$ CYP2E1 overexpression in breast cancer cells is involved in the alteration of numerous pathways linked to the disease such as cell cycle control, apoptosis, autophagy, ER stress and UPR. ${ }^{[2,44,45]}$ In addition to these, another pathway that is regulated by the cellular redox state is cellular energy metabolism. The interrelation between ROS and aerobic glycolysis which is the main pathway through which cancer cells produce energy has been extensively investigated. ${ }^{[31,32]}$ Since cytochrome P450 enzymes are one of the endogenous sources of $\operatorname{ROS}^{[46,47]}$ it was hypothesized that CYP450s might be involved in the regulation of cellular energy metabolism.

The role of the CYP2E1 mediated ROS generation in the energy metabolism of breast cancer cells was investigated in the estrogen receptor positive MCF-7 and estrogen receptor negative MDA-MB-231 cells. ${ }^{[48]}$ Given that CYP2E1 gene expression is under the transcriptional control of factors responsive to inflammation ${ }^{[1,29,49,50]}$ it was theorized that inhibition of glycolysis in breast cancer cells bearing diverse genetic background would lead to alternative CYP2E1 cellular levels and hence dissimilar ROS. ${ }^{[42]}$ On the other side inhibition of glycolysis would lead to increased ROS generation ${ }^{[31]}$ that could be altered by CYP2E1 enzymatic activity. ${ }^{[42,51-53]}$ In accord with published results treatment of both MCF-7 and MDAMB-231 cells with the glycolytic inhibitors 3BP and 2DG increased ROS levels in the two cell lines. ${ }^{[33,54,55]}$ Combination of either 3BP or 2DG with the CYP2E1 inhibitor CMZ reduced dramatically the oxygen radicals' levels in the MCF-7 but not in the MDA-MB-231 cells [Figure 2] implying that the dissimilar genetic background in the two cell lines (wild type ER and p53 in MCF-7 and defective ER and mutated p53 in MDA-MB-231 cells) determines the differential response of these cells to the glycolytic and CYP2E1 inhibitors. ${ }^{[6]}$

The observation that CMZ decreased ROS generation stimulated by 3BP and 2DG treatment in MCF-7 cells prompted our interest to explore the possibility that CYP2E1 is involved in the process of energy metabolism in breast cancer cells. The potential link between CYP2E1 and energy metabolism was investigated in the MCF-7 and MDA-MB-231 cells by estimating the ATP production after treating these cells with the CYP2E1 inhibitor CMZ. Results shown in Figure 3 indicate that CMZ treatment did not significantly affect ATP generation in the two cell lines implying that if CYP2E1 had inhibitory effect on one of the pathways of ATP generation another pathway compensates for the loss facilitating cells to meet their energy requirements, ${ }^{[57]}$ or CYP2E1 is not involved in ATP production in these cells. To answer these questions the lactate concentration was determined in CMZ treated MCF-7 and MDA-MB-231 cells. ${ }^{[58]}$ CMZ treatment reduced lactate efflux in MCF-7 but not in MDA-MB-231 cells [Figure 3C and 3D] implying that CYP2E1 exerts cell type dependent effects on energy metabolism. These results are in line with those shown in Figure 2 indicating reversion of the effect of the glycolytic inhibitors 3BP and 2DG on ROS levels by CMZ in MCF-7 cells, and published studies reporting that high ROS levels induce hypoxia inducible factor 1 alpha (HIF-1 $\alpha$ ) thereby 
inducing lactate dehydrogenase (LDH-A) gene expression and hence lactate efflux. ${ }^{[58]}$

Depolarization of the mitochondrial membrane is determined by the gradient of protons across the mitochondrial membrane. Opening of the mitochondrial permeability transition pore (PTP) permitting influx or efflux of protons can lead to mitochondrial membrane depolarization. Proteins involved in the regulation of the PTP opening are susceptible to redox modifications therefore high levels of ROS may lead to PTP opening and induce mitochondrial membrane depolarization. ${ }^{[59]}$ Given that overexpression [Figure 1] or inhibition of CYP2E1 altered the redox state of MCF-7 and MDA-MB-231 cells [Figure 2] we were interested to study potential changes of the mitochondrial membrane potential in breast cancer cells attributed to the activation or inhibition of the CYP2E1 enzymatic activity. Reduced mitochondrial membrane potential was observed in both MCF-7 and MDA-MB-231 cells treated with the CYP2E1 activator APAP whereas no changes in mitochondrial membrane depolarization were recorded in these cells treated with the CYP2E1 inhibitor CMZ [Figure 4].

Cell death through the intrinsic pathway of apoptosis is triggered by sustained mitochondrial membrane depolarization. ${ }^{[59]}$ To investigate the potential role of CYP2E1 in inducing cell death by mediating alterations in the mitochondrial membrane depolarization MCF-7 and MDA-MB-231 cells were treated with either the CYP2E1 activator APAP or the CYP2E1 inhibitor CMZ and cellular viability was assessed. APAP induced cell death in both MCF-7 and MDA-MB-231 cells while CMZ induced cell death only in MCF-7 cells [Figure 5] reiterating the view that CYP2E1 effects are cell type specific.

Taken together results presented in this study provide evidence to support the concept that CYP2E1 regulates cellular energy metabolism in a cell type dependent manner affecting predominately this pathway in less invasive and early stages of breast cancer represented by the MCF-7 cells. Although these results require validation in an in vivo system they endorse the conclusion that CYP2E1 cellular levels can be a prognostic indicator and a potential breast cancer biomarker.

\section{Financial support and sponsorship}

SS and CD are supported by the Manchester Pharmacy School, University of Manchester.

\section{Conflicts of interest}

There are no conflicts of interest.

\section{REFERENCES}

1. Lenaz G, Baracca A, Barbero G, Bergamini C, Dalmonte ME, Del Sole M, Faccioli M, Falasca A, Fato R, Genova ML, Sgarbi G, Solaini G. Mitochondrial respiratory chain super-complex I-III in physiology and pathology. Biochim Biophys Acta 2010;1797:633-40.

2. Schieber M, Chandel Navdeep S. ROS Function in Redox Signaling and Oxidative Stress. Current Biology 2014;24:R453-62.

3. Burhans WC, Heintz NH. The cell cycle is a redox cycle: linking phase-specific targets to cell fate. Free Radic Biol Med 2009; 47:1282-93

4. Dewaele M, Maes H, Agostinis P. ROS-mediated mechanisms of autophagy stimulation and their relevance in cancer therapy. Autophagy 2010;6:838-54.

5. Azad MB, Chen Y, Gibson SB. Regulation of autophagy by reactive oxygen species (ROS): implications for cancer progression and treatment. Antioxid Redox Signal 2009;11:777-90.

6. Wu WS. The signaling mechanism of ROS in tumor progression. Cancer Metastasis Rev 2006;25:695-705.

7. Bhandary B, Marahatta A, Kim HR, Chae HJ. An involvement of oxidative stress in endoplasmic reticulum stress and its associated diseases. Int J Mol Sci 2012;14:434-56.

8. Sullivan LB, Chandel NS. Mitochondrial reactive oxygen species and cancer. Cancer Metab 2014;2:17.

9. Quijano C, Trujillo M, Castro L, Trostchansky A. Interplay between oxidant species and energy metabolism. Redox biology 2016;8:28-42.

10. Holmstrom KM, Finkel T. Cellular mechanisms and physiological consequences of redox-dependent signalling. Nat Rev Mol Cell Biol 2014; 15:411-21.

11. Coon MJ. Cytochrome P450: nature's most versatile biological catalyst. Аnпu Rev Pharmacol Toxicol 2005;45:1-25.

12. Hrycay EG, Bandiera SM. Monooxygenase, peroxidase and peroxygenase properties and reaction mechanisms of cytochrome P450 enzymes. Adv Exp Med Biol 2015;851:1-61.

13. Gonzalez FJ. Role of cytochromes P450 in chemical toxicity and oxidative stress: studies with CYP2E1. Mutat Res 2005;569:101-10.

14. Tamasi V, Monostory K, Prough RA, Falus A. Role of xenobiotic metabolism in cancer: involvement of transcriptional and miRNA regulation of P450s. Cell Mol Life Sci 2011;68:1131-46.

15. McFadyen MC, Melvin WT, Murray GI. Cytochrome P450 enzymes: novel options for cancer therapeutics. Mol Cancer Ther 2004;3:363-71.

16. Iscan M, Klaavuniemi T, Coban T, Kapucuoglu N, Pelkonen O, Raunio H. The expression of cytochrome P450 enzymes in human breast tumours and normal breast tissue. Breast Cancer Res Treat 2001;70:47-54.

17. Kapucuoglu N, Coban T, Raunio H, Pelkonen O, Edwards RJ, Boobis AR, Iscan M. Immunohistochemical demonstration of the expression of CYP2E1 in human breast tumour and non-tumour tissues. Cancer Lett 2003;196:153-9.

18. Morgan ET, Goralski KB, Piquette-Miller M, Renton KW, Robertson GR, Chaluvadi MR, Charles KA, Clarke SJ, Kacevska M, Liddle C, Richardson TA, Sharma R, Sinal CJ. Regulation of drugmetabolizing enzymes and transporters in infection, inflammation, and cancer. Drug Metab Dispos 2008;36:205-16.

19. Zordoky BN, El-Kadi AO. Role of NF-kappaB in the regulation of cytochrome P450 enzymes. Curr Drug Metab 2009;10:164-78.

20. Wang J, Hu Y, Nekvindova J, Ingelman-Sundberg M, Neve EP. IL-4-mediated transcriptional regulation of human CYP2E1 by two independent signaling pathways. Biochem Pharmacol 2010;80:1592-600.

21. Millonig G, Wang Y, Homann N, Bernhardt F, Qin H, Mueller S, Bartsch H, Seitz HK. Ethanol-mediated carcinogenesis in the human esophagus implicates CYP2E1 induction and the generation of carcinogenic DNA-lesions. Int J Cancer 2011;128:533-40.

22. Wang S, Sugamori KS, Tung A, McPherson JP, Grant DM. N-hydroxylation of 4-aminobiphenyl by CYP2E1 produces oxidative stress in a mouse model of chemically induced liver cancer. Toxicol Sci 2015;144:393-405.

23. Kang JS, Wanibuchi H, Morimura K, GonzalezFJ, Fukushima S. Role of CYP2E1 in diethylnitrosamine-induced hepatocarcinogenesis in 
vivo. Cancer Res 2007;67:11141-6.

24. Miller TW, Isenberg JS, Roberts DD. Molecular regulation of tumor angiogenesis and perfusion via redox signaling. Chem Rev 2009;109:3099-124.

25. Scherz-Shouval R, Elazar Z. Regulation of autophagy by ROS: physiology and pathology. Trends Biochem Sci 2011;36:30-8.

26. Ravikumar B, Sarkar S, Davies JE, Futter M, Garcia-Arencibia M, Green-Thompson ZW, Jimenez-Sanchez M, Korolchuk VI, Lichtenberg M, Luo S, Massey DC, Menzies FM, Moreau K, Narayanan U, Renna M, Siddiqi FH, Underwood BR, Winslow AR, Rubinsztein DC. Regulation of mammalian autophagy in physiology and pathophysiology. Physiol Rev 2010;90:1383-435.

27. Kim I, Xu W, Reed JC. Cell death and endoplasmic reticulum stress: disease relevance and therapeutic opportunities. Nat Rev Drug Discov 2008;7:1013-30.

28. Rutkowski DT, Kaufman RJ. A trip to the ER: coping with stress. Trends Cell Biol 2004;14:20-8.

29. Leung T, Rajendran R, Singh S, Garva R, Krstic-Demonacos M, Demonacos C. Cytochrome P450 2E1 (CYP2E1) regulates the response to oxidative stress and migration of breast cancer cells. Breast Cancer Res 2013;15:R107.

30. Pavlova NN, Thompson CB. The emerging hallmarks of cancer metabolism. Cell Metab 2016;23:27-47.

31. Liemburg-Apers DC, Willems PH, Koopman WJ, Grefte S. Interactions between mitochondrial reactive oxygen species and cellular glucose metabolism. Arch Toxicol 2015;89:1209-26.

32. Kang SW, Lee S, Lee EK. ROS and energy metabolism in cancer cells: alliance for fast growth. Arch Pharm Res 2015;38:338-45.

33. El Sayed SM, Mahmoud AA, El Sawy SA, Abdelaal EA, Fouad AM, Yousif RS, Hashim MS, Hemdan SB, Kadry ZM, Abdelmoaty MA, Gabr AG, Omran FM, Nabo MM, Ahmed NS. Warburg effect increases steady-state ROS condition in cancer cells through decreasing their antioxidant capacities (anticancer effects of 3-bromopyruvate through antagonizing Warburg effect). Med Hypotheses 2013;81:866-70.

34. Vaclavikova R, Hubackova M, Stribrna-Sarmanova J, Kodet R, Mrhalova M, Novotny JAN, Gut I, Soucek P. RNA expression of cytochrome P450 in breast cancer patients. Anticancer Res 2007;27:4443-50.

35. Zheng J. Energy metabolism of cancer: Glycolysis versus oxidative phosphorylation (Review). Oncol Lett 2012;4:1151-7.

36. Hu Y, Mishin V, Johansson I, von Bahr C, Cross A, Ronis MJ, Badger TM, Ingelman-Sundberg $M$. Chlormethiazole as an efficient inhibitor of cytochrome P450 2E1 expression in rat liver. $J$ Pharmacol Exp Ther 1994;269:1286-91.

37. Pelkonen O, Turpeinen M, Hakkola J, Honkakoski P, Hukkanen J, Raunio H. Inhibition and induction of human cytochrome P450 enzymes: current status. Arch Toxicol 2008;82:667-715.

38. Zorov DB, Juhaszova M, Sollott SJ. Mitochondrial reactive oxygen species (ROS) and ROS-induced ROS release. Physiol Rev 2014;94:909-50.

39. Kroemer G, Galluzzi L, Brenner C. Mitochondrial Membrane Permeabilization in Cell Death. Physiol Rev 2007;87:99-163.

40. Schattenberg JM, Czaja MJ. Regulation of the effects of CYP2E1induced oxidative stress by JNK signaling. Redox biology 2014;3:7-15.

41. Raza H, Prabu SK, Robin MA, Avadhani NG. Elevated mitochondrial cytochrome P450 2E1 and glutathione S-transferase A4-4 in streptozotocin-induced diabetic rats: tissue-specific variations and roles in oxidative stress. Diabetes 2004;53:185-94.

42. Lvdan, Wei Z, Baodan, Xiongzhi F, Huang L, Lianfeng Z.
Downregulation of CYP2E1 ameliorates oxidative stress and apoptosis. Heart 2011;97:A69.

43. Jin M, Ande A, Kumar A, Kumar S. Regulation of cytochrome P450 2e1 expression by ethanol: role of oxidative stress-mediated pkc/ jnk/sp1 pathway. Cell Death Dis 2013;4:e554.

44. Lewis MD, Roberts BJ. Role of CYP2E1 activity in endoplasmic reticulum ubiquitination, proteasome association, and the unfolded protein response. Arch Biochem Biophys 2005;436:237-45.

45. Nebert DW, Dalton TP. The role of cytochrome P450 enzymes in endogenous signalling pathways and environmental carcinogenesis. Nat Rev Cancer 2006;6:947-60.

46. Zangar RC, Davydov DR, Verma S. Mechanisms that regulate production of reactive oxygen species by cytochrome P450. Toxicol Appl Pharmacol 2004;199:316-31.

47. Namazi MR. Cytochrome-P450 enzymes and autoimmunity: expansion of the relationship and introduction of free radicals as the link. J Autoimmune Dis 2009;6:4

48. Nizamutdinova IT, Lee GW, Son KH, Jeon SJ, Kang SS, Kim YS, Lee JH, Seo HG, Chang KC, Kim HJ. Tanshinone I effectively induces apoptosis in estrogen receptor-positive (MCF-7) and estrogen receptor-negative (MDA-MB-231) breast cancer cells. Int J Oncol 2008;33:485-91.

49. Kim DK, Kim YH, Jang HH, Park J, Kim JR, Koh M, Jeong WI, Koo SH, Park TS, Yun CH, Park SB, Chiang JY, Lee CH, Choi HS. Estrogen-related receptor gamma controls hepatic CB1 receptormediated CYP2E1 expression and oxidative liver injury by alcohol. Gut 2013;62:1044-54.

50. Aitken AE, Richardson TA, Morgan ET. Regulation of drugmetabolizing enzymes and transporters in inflammation. Annu Rev Pharmacol Toxicol 2006;46:123-49.

51. Das J, Ghosh J, Manna P, Sil PC. Taurine protects acetaminopheninduced oxidative damage in mice kidney through APAP urinary excretion and CYP2E1 inactivation. Toxicology 2010;269:24-34.

52. Das J, Ghosh J, Manna P, Sil PC. Acetaminophen induced acute liver failure via oxidative stress and JNK activation: protective role of taurine by the suppression of cytochrome P450 2E1. Free Radic Res 2010;44:340-55.

53. Cederbaum A. Nrf2 and antioxidant defense against CYP2E1 toxicity. Expert Opin Drug Metab Toxicol 2009;5:1223-44.

54. Shutt DC, O'Dorisio MS, Aykin-Burns N, Spitz DR. 2-deoxyD-glucose induces oxidative stress and cell killing in human neuroblastoma cells. Cancer Biol Ther 2010;9:853-61.

55. Ihrlund LS, Hernlund E, Khan O, Shoshan MC. 3-Bromopyruvate as inhibitor of tumour cell energy metabolism and chemopotentiator of platinum drugs. Mol Oncol 2008;2:94-101.

56. Wickramasekera NT, Das GM. Tumor suppressor p53 and estrogen receptors in nuclear-mitochondrial communication. Mitochondrion 2014;16:26-37.

57. Rodriguez-Enriquez S, Carreno-Fuentes L, Gallardo-Perez JC, Saavedra E, Quezada H, Vega A, Marin-Hernandez A, OlinSandoval V, Torres-Marquez ME, Moreno-Sanchez R. Oxidative phosphorylation is impaired by prolonged hypoxia in breast and possibly in cervix carcinoma. Int $J$ Biochem Cell Biol 2010;42:1744-51.

58. Doherty JR, Cleveland JL. Targeting lactate metabolism for cancer therapeutics. J Clin Invest 2013;123:3685-92.

59. Echtay KS, Roussel D, St-Pierre J, Jekabsons MB, Cadenas S, Stuart JA, Harper JA, Roebuck SJ, Morrison A, Pickering S, Clapham JC, Brand MD. Superoxide activates mitochondrial uncoupling proteins. Nature 2002;415:96-9. 